\title{
Reliability Optimum Design for Bevel Gear Driven Systems Based on Genetics Algorithm
}

\author{
Chang $\mathrm{Li}^{\mathrm{a},}{ }^{*}$, Mingyong $\mathrm{Hu}^{\mathrm{b}}$ and Xing Han ${ }^{\mathrm{c}}$ \\ School of Mechanical Engineering \& Automation, University of Science and Technology Liaoning, \\ Anshan 114051, China. \\ alichang2323-23@163.com, b2277182899@qq.com, chanxinggctx@163.com
}

\begin{abstract}
Genetics algorithm is a global group search optimum calculation method based on the natural selections and the genetic variations. Mechanic reliability design makes sure that the reliability indexes can be realized. However, it cannot guarantee that the products have the best working performances and the optimum design parameters. In this paper, genetics algorithm is introduced into the reliability optimum design for bevel gear driven systems, and then the optimum design parameters of driven systems are obtained under the allowable reliability degree. This method can quantificational make optimum analysis for bevel gear driven systems, and is very useful for the engineering application.
\end{abstract}

Keywords: Bevel gear driven systems; reliability optimum design; random parameters; genetics algorithm.

\section{Introduction}

With the development of modern industry and machinery science technology, the bevel gear driven system is required to have the characteristics of light-duty, high-speed, large carrying power, long life and high reliability [1-3]. In the era of rapid development computer, compared with the actual situation, the accuracy of calculation parameters is far behind the precise analysis of engineering structures. Therefore, considering the random factors of actual situation have a very significant impact on the reliability optimum design for bevel gear driven systems based on genetics algorithm.

Genetics algorithm describes problems by a single string, makes optimum calculation by using the fitness function without regard to the gradient information such as functional derivatives, etc. Therefore, it is good at solving the complex nonlinear problems that other subjects don't solve or are difficult to solve. Reliability optimum design for bevel gear driven systems based on genetics algorithm can guarantee that the driven systems have the best working performances and the optimum design parameters.

\section{Establishing Reliability Optimum Mathematical Model for Bevel Gear Driven Systems}

\subsection{Establishing Optimum Objective Function.}

The least all-in cost of bevel gear driven system design is set for the objective function. It can be written as:

$\min C_{T}=C_{1}\left(\mu_{\delta}\right)+C_{2}\left(\sigma_{\delta}\right)+C_{3}\left(\mu_{s}\right)+C_{4}\left(\sigma_{s}\right)$

Where, $C_{T}$ is the all-in cost, $C_{1}\left(\mu_{\delta}\right)$ and $C_{2}\left(\sigma_{\delta}\right)$ are respectively the cost functions of strength equalizing value and strength standard deviation, $C_{3}\left(\mu_{s}\right)$ and $C_{4}\left(\sigma_{s}\right)$ are respectively the cost functions of stress equalizing value and stress standard deviation.

In many factors, the cost of bevel gear material is an important factor for the all-in cost. Therefore, the cost of material is mainly taken into consideration. $C_{T}$ is set for:

$C_{T}=\left(V_{1}+V_{2}\right) \rho$

Where, $\rho$ is the material density of bevel gear, $V_{1}$ and $V_{2}$ are respectively the volumes of driving gear and driven gear. The bevel gear drive mechanism is shown in Fig.1. The gear mesh volumes of driving gear and driven gear are respectively obtained, as shown in Eqs.1 and 2. 


$$
\begin{aligned}
& V_{1}=\frac{\pi}{3} b \cos \delta_{1} \times\left[\left(\frac{m z_{1}}{2}\right)^{2}+\frac{m z_{1}}{2}\left(\frac{R-b}{R} \times \frac{m z_{1}}{2}\right)+\left(\frac{R-b}{R} \times \frac{m z_{1}}{2}\right)^{2}\right] \\
& V_{2}=\frac{\pi}{3} b \cos \delta_{2} \times\left[\left(\frac{m z_{2}}{2}\right)^{2}+\frac{m z_{2}}{2}\left(\frac{R-b}{R} \times \frac{m z_{2}}{2}\right)+\left(\frac{R-b}{R} \times \frac{m z_{2}}{2}\right)^{2}\right]
\end{aligned}
$$

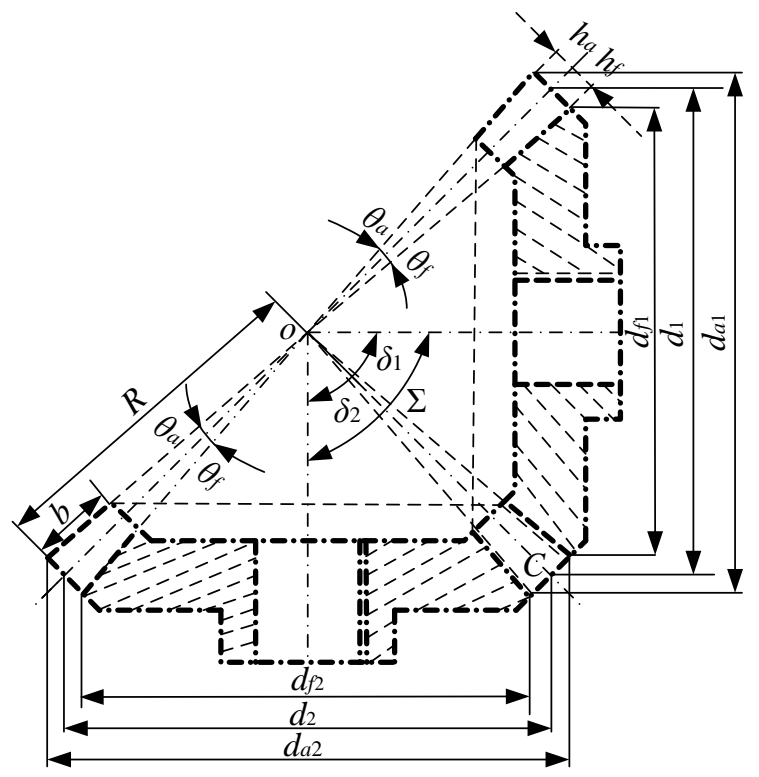

Fig. 1 Driven structure of orthogonal bevel gears

Where, $z_{1}$ and $z_{2}$ are respectively the tooth numbers of driving gear and driven gear; $m$ is the big end module; $R$ is the cone distance; $b$ is the tooth width; $\delta_{1}$ and $\delta_{2}$ are respectively the reference cone angles of driving gear and driven gear.

\subsection{Determining Reliability Optimum Constraints.}

The contact stresses of driving gear and driven gear are calculated respectively. We selected the smaller value. The contact stress of bevel gear is obtained by Eq. 3 .

$$
\sigma_{H}=Z_{H} Z_{E} Z_{\varepsilon} Z_{\beta} Z_{k} \sqrt{\frac{K_{A} K_{V} K_{H \beta} K_{H \alpha} F_{t m}}{d_{m 1} b_{e H}} \times \frac{\sqrt{u^{2}+1}}{u}}
$$

Where, $u$ is the gear ratio; $Z_{H}$ is the node region coefficient; $Z_{E}$ is the elastic coefficient and we set $Z_{E}=189.8 \sqrt{\mathrm{N} / \mathrm{mm}^{2}} ; Z_{\varepsilon}$ is the overlap ratio coefficient and we set $Z_{\varepsilon}=\sqrt{\left(4-\varepsilon_{v \alpha}\right) / 3} ; Z_{\beta}$ is the helix angle coefficient of contact strength calculation; we set $Z_{k}=0.85 ; K_{A}$ is the utilization coefficient; $K_{V}$ is the dynamic load factor; $K_{H \beta}$ is the distribution coefficient of longitudinal form load; $K_{H \alpha}$ is the partition ratio of longitudinal form load; $F_{t m}$ is the nominal shear stress on pitch circle of tooth width midpoint; $d_{m 1}$ is the pitch circle diameter of virtual cylindrical gear of bevel pinion; $b_{e H}$ is the effective tooth width and we set $b_{e H}=0.85 b ; u$ is the gear ratio.

We selected the equalizing values of all parameters in Eq. 3. Therefore, the contact stress equalizing value of bevel gear $\bar{\sigma}_{H}$ is determined. Meanwhile, according to the literature [2], the variation coefficient of contact stress can be written as [4-6]:

$$
C_{\sigma_{H}}=\left[C_{z_{H}}^{2}+C_{z_{E}}^{2}+C_{z_{\varepsilon}}^{2}+C_{z_{\beta}}^{2}+\frac{1}{4}\left(C_{F_{m}}^{2}+C_{d_{m 1}}^{2}+C_{b_{e H}}^{2}+C_{K_{A}}^{2}+C_{K_{V}}^{2}+C_{K_{H \beta}}^{2}+C_{K_{H \alpha}}^{2}\right)\right]^{1 / 2}
$$

The standard deviation of contact stress is:

$$
s_{\sigma_{H}}=C_{\sigma_{H}} \times \bar{\sigma}_{H}
$$

The allowable contact stress is:

$$
\sigma_{H p}=\frac{\sigma_{H \lim }}{S_{H \text { min }}} Z_{L} Z_{V} Z_{R} Z_{X}
$$

Where, $\sigma_{H \mathrm{Him}}$ is the contact fatigue strength limit of experimental gear; $S_{H \min }$ is the least safety factor of contact strength calculation; $Z_{L}$ is the lubricant coefficient; $Z_{V}$ is the velocity coefficient; $Z_{R}$ 
is the coefficient of rugosity; $Z_{X}$ is the dimension coefficient of contact strength calculation; The above parameters can be determined by the corresponding forms of literature [1].

We selected the equalizing values of all parameters in Eq. 6. Therefore, the allowable contact stress equalizing value of bevel gear $\bar{\sigma}_{H p}$ is determined. Meanwhile, according to the literature [2], the variation coefficient of allowable contact stress can be written as:

$$
C_{\sigma_{H p}}=\left[C_{\sigma_{H \text { lim }}}^{2}+C_{S_{H \text { in }}}^{2}+C_{Z_{L}}^{2}+C_{Z_{V}}^{2}+C_{Z_{R}}^{2}+C_{Z_{X}}^{2}\right]^{1 / 2}
$$

The standard deviation of allowable contact stress is:

$$
s_{\sigma_{H p}}=C_{\sigma_{H p}} \times \bar{\sigma}_{H p}
$$

According to the above conditions, the reliability optimum constraint of contact stress can be determined as:

$$
G_{1}(x)=R_{H}-\left[R_{H}\right] \geq 0
$$

Suppose stress and strength all conform to the normal distribution, the constraint can be written as:

$$
z_{R_{H}}=\frac{\mu_{\sigma_{H p}}-\mu_{\sigma_{H}}}{\sqrt{\sigma_{\sigma_{H p}}{ }^{2}+\sigma_{\sigma_{H}}{ }^{2}}} \geq z_{\left[R_{H}\right]}
$$

In the same way, the dedendum flexural stress of bevel gear can be written as:

$$
\sigma_{F}=\frac{K_{A} K_{V} K_{F \beta} K_{F \alpha} F_{t m}}{b_{e F} m_{n m}} Y_{F a} Y_{S a} Y_{\varepsilon} Y_{\beta} Y_{k}
$$

Where, $K_{A}$ is the utilization coefficient; $K_{V}$ is the dynamic load factor; $K_{F \beta}$ is the distribution coefficient of longitudinal form load of flexural strength calculation; $K_{F \alpha}$ is the partition ratio of tooth space load; $F_{t m}$ is the nominal shear stress on pitch circle of tooth width midpoint; we set $b_{e F}=$ $0.85 b ; m_{n m}$ is the normal module of tooth width midpoint; $Y_{F a}$ is the stress correction factor; $Y_{\varepsilon}$ is the overlap ratio coefficient; $Y_{\beta}$ is the helix angle coefficient; we set $Y_{k}=1$.

We selected the equalizing values of all parameters in Eq. 10. Therefore, the dedendum flexural stress equalizing value of bevel gear $\bar{\sigma}_{F}$ is determined. Meanwhile, according to the literature [2], the variation coefficient of dedendum flexural stress can be written as:

$$
C_{\sigma_{F}}=\left[C_{K_{A}}^{2}+C_{K_{V}}^{2}+C_{K_{F \beta}}^{2}+C_{K_{F \alpha}}^{2}+C_{F_{m m}}^{2}+C_{b_{e F}}^{2}+C_{m_{n m}}^{2}+C_{Y_{F a}}^{2}+C_{Y_{s a}}^{2}+C_{Y_{\varepsilon}}^{2}+C_{Y_{\beta}}^{2}+C_{Y_{k}}^{2}\right]^{1 / 2}
$$

The standard deviation of dedendum flexural stress is:

$$
s_{\sigma_{F}}=C_{\sigma_{F}} \times \bar{\sigma}_{F}
$$

The allowable dedendum flexural stress is:

$$
\sigma_{F p}=\frac{\sigma_{F \lim } Y_{S T}}{S_{F \min }} Y_{\text {סrelT }} Y_{\text {RrelT }} Y_{X}
$$

Where, $\sigma_{\text {Flim }}$ is the bending fatigue limit of experimental gear; $Y_{S T}$ is the stress correction factor of experimental gear and we set $Y_{S T}=2.0 ; S_{F \min }$ is the least safety factor of flexural strength calculation; $Y_{\delta r e l T}$ is the sensitivity coefficient of relative tooth root fillet; $Y_{\text {RrelT }}$ is the condition coefficient of relative heel of tooth; $Y_{X}$ is the dimension coefficient of flexural strength calculation; The above parameters can be determined by the corresponding forms of literature [1].

We selected the equalizing values of all parameters in Eq. 12. Therefore, the allowable dedendum flexural stress equalizing value $\bar{\sigma}_{F p}$ is determined. Meanwhile, according to the literature [2], the variation coefficient of allowable dedendum flexural stress can be written as:

$$
C_{\sigma_{F p}}=\left[C_{\sigma_{F \text { Iim }}}^{2}+C_{Y_{S T}}^{2}+C_{S_{F \min }}^{2}+C_{Y_{\text {Oert }}}^{2}+C_{Y_{\text {Rert }}}^{2}+C_{Y_{X}}^{2}\right]^{1 / 2}
$$

The standard deviation of allowable dedendum flexural stress is:

$$
s_{\sigma_{F p}}=C_{\sigma_{F p}} \times \bar{\sigma}_{F p}
$$

According to the above conditions, the reliability optimum constraint of dedendum flexural stress can be determined as: 


$$
G_{2}(x)=R_{F}-\left[R_{F}\right] \geq 0
$$

Suppose stress and strength all conform to the normal distribution, the constraint can be written as:

$$
z_{R_{F}}=\frac{\mu_{\sigma_{H p}}-\mu_{\sigma_{H}}}{\sqrt{\sigma_{\sigma_{H p}}^{2}+\sigma_{\sigma_{H}}^{2}}} \geq z_{\left[R_{F}\right]}
$$

\subsection{Design Variables and Edge-restraint Conditions.}

The tooth number of driving bevel gear $z_{1 \max } \geq z_{1} \geq 17 \cos \delta_{1}$; the module of gear pair $m \geq 2$; the tooth width coefficient of gear pair $0.35 \geq \psi_{b} \geq 0.2$; the density of gear material $\rho \geq 0$.

\section{Making Fitness Function with Punishment Function Method}

According to the exterior point punishment function method, we made the fitness function for the constraint nonlinear programming problem, as follows [7-9]:

$\operatorname{Val}(x)=f(x)+L(x)$

Where, $x$ represents the chromosome; $f(x)$ is the objective function; $L(x)$ is the penalty term.

For the minimum value, we have:

$\left\{\begin{array}{l}L(x)=0, \text { the feasible } x \\ L(x)=-s_{1}\left[G_{1}(x)\right]^{2}-s_{2}\left[G_{2}(x)\right]^{2}<0, \text { the infeasible } x\end{array}\right.$

Where, $s_{1}$ and $s_{2}$ are respectively the penalty factors of two inequality constraint functions. According to the design criteria of encased gear, we set $s_{1}=1$ and $s_{2}=0.5$, respectively. We regarded Eqs. 9 and 11 as the penalty terms in the fitness function. Meanwhile, we regarded the boundary conditions of design variables as the matrix of variables top and bottom limitation in the genetics algorithm.

\section{Model Solution of Reliability Optimum Design for Bevel Gears Based on Genetics Algorithm}

In calculation, we selected the orthogonal $90^{\circ}$ encased straight bevel gear driven system as the optimum design object. Meanwhile, we set that the gear-driven useful horsepower is $9.8 \mathrm{~kW}$; the speed of driving gear is $1000 \mathrm{r} / \mathrm{min}$; the gear ratio is 3; the working condition coefficient $K$ is 1.8 ; the material of small bevel gear is $40 \mathrm{Cr}$, modified treatment, the rigidity is $250 \mathrm{HBS}$; the material of big bevel gear is $35 \mathrm{SiMnA}$, modified treatment, the rigidity is $230 \mathrm{HBS}$; the densities of two bevel gears are set according to their materials; the reliability of allowable contact stress $\left[R_{H}\right]$ and dedendum flexural stress $\left[R_{F}\right]$ are respectively 0.9999 .

According to the above calculation conditions, we wrote the programs by MATLAB. By calling the genetics search function and iterative computations, the least optimum result of all-in cost of bevel gear driven system design is obtained.

Table 1 shows the data of search path of population optimum. Fig. 2 shows the trend curve of average changes of each generation optimal sufficiency in genetics algorithm. 
Table 1 Search path data of bevel gear pair reliability optimum based on genetics algorithm

\begin{tabular}{|c|c|c|c|c|c|c|c|c|c|}
\hline $\begin{array}{l}\text { hereditary } \\
\text { algebra }\end{array}$ & $\begin{array}{c}\text { tooth number } \\
\text { of small bevel } \\
\text { gear }\end{array}$ & modulo/mm & $\begin{array}{l}\text { coefficient of } \\
\text { face width }\end{array}$ & $\begin{array}{l}\text { mass of bevel gear } \\
\text { driven system } / \mathrm{kg}\end{array}$ & $\begin{array}{l}\text { Hereditary } \\
\text { algebra }\end{array}$ & $\begin{array}{c}\text { tooth number } \\
\text { of small bevel } \\
\text { gear }\end{array}$ & modulo/mm & $\begin{array}{l}\text { coefficient of } \\
\text { face width }\end{array}$ & $\begin{array}{l}\text { mass of bevel gear } \\
\text { driven system } / \mathrm{kg}\end{array}$ \\
\hline 1 & 28.0231186647 & 2.04467151358 & 0.275670035500 & 1.438088031306750 & 53 & 18.7297380165 & 2.00000000000 & 0.250000000000 & 0.374710945232760 \\
\hline 3 & 17.2836767226 & 2.00000000000 & 0.271111329306 & 0.604524556291095 & 54 & 18.7288391897 & 2.00000000000 & 0.250000000000 & 0.374657001485795 \\
\hline 4 & 19.8973428501 & 2.00000000000 & 0.274347885569 & 0.480150955772500 & 55 & 18.7287417624 & 2.00000000000 & 0.250000000000 & 0.374651154623675 \\
\hline 5 & 19.1833249969 & 2.00000000000 & 0.270891590979 & 0.426472536958825 & 57 & 18.7286519706 & 2.00000000000 & 0.250000000000 & 0.374645766042985 \\
\hline 7 & 19.2572809131 & 2.00000000000 & 0.250000000000 & 0.407273514076675 & 60 & 18.7286485740 & 2.00000000000 & 0.250000000000 & 0.374645562211455 \\
\hline 8 & 18.8180708343 & 2.00000000000 & 0.270891590979 & 0.402573063923525 & 63 & 18.7286474356 & 2.00000000000 & 0.250000000000 & 0.374645493892905 \\
\hline 10 & 19.0285888848 & 2.00000000000 & 0.250234992208 & 0.393204715819815 & 66 & 18.7286472062 & 2.00000000000 & 0.250000000000 & 0.374645480124005 \\
\hline 11 & 18.7516101421 & 2.00000000000 & 0.250000000000 & 0.376025213372810 & 67 & 18.7285908895 & 2.00000000000 & 0.250000000000 & 0.374642100479205 \\
\hline 16 & 18.7516024263 & 2.00000000000 & 0.250000000000 & 0.376024749203095 & 69 & 18.7285514548 & 2.00000000000 & 0.250000000000 & 0.374639733954620 \\
\hline 18 & 18.7515983265 & 2.00000000000 & 0.250000000000 & 0.376024502563945 & 70 & 18.7285486653 & 2.00000000000 & 0.250000000000 & 0.374639566554155 \\
\hline 19 & 18.7515954574 & 2.00000000000 & 0.250000000000 & 0.376024329962070 & 71 & 18.7285155871 & 2.00000000000 & 0.250000000000 & 0.374637581502675 \\
\hline 21 & 18.7515934792 & 2.00000000000 & 0.250000000000 & 0.376024210952145 & 73 & 18.7285034656 & 2.00000000000 & 0.250000000000 & 0.374636854087135 \\
\hline 22 & 18.7494590795 & 2.00000000000 & 0.250000000000 & 0.375895822728700 & 75 & 18.7284934782 & 2.00000000000 & 0.250000000000 & 0.374636254732570 \\
\hline 23 & 18.7462003196 & 2.00000000000 & 0.250000000000 & 0.375699858457710 & 77 & 18.7284893052 & 2.00000000000 & 0.250000000000 & 0.374636004308935 \\
\hline 25 & 18.7458119773 & 2.00000000000 & 0.250000000000 & 0.375676510185440 & 79 & 18.7284868680 & 2.00000000000 & 0.250000000000 & 0.374635858054800 \\
\hline 28 & 18.7434166926 & 2.00000000000 & 0.250000000000 & 0.375532520072865 & 80 & 18.7284858998 & 2.00000000000 & 0.250000000000 & 0.374635799953025 \\
\hline 30 & 18.7422694076 & 2.00000000000 & 0.250000000000 & 0.375463565225415 & 81 & 18.7284856693 & 2.00000000000 & 0.250000000000 & 0.374635786115830 \\
\hline 31 & 18.7415117725 & 2.00000000000 & 0.250000000000 & 0.375418033995320 & 82 & 18.7284856174 & 2.00000000000 & 0.250000000000 & 0.374635783002520 \\
\hline 33 & 18.7414408156 & 2.00000000000 & 0.250000000000 & 0.375413769921635 & 83 & 18.7284856016 & 2.00000000000 & 0.250000000000 & 0.374635782053455 \\
\hline 35 & 18.7414279650 & 2.00000000000 & 0.250000000000 & 0.375412997684165 & 84 & 18.7284855993 & 2.00000000000 & 0.250000000000 & 0.374635781920005 \\
\hline 38 & 18.7406091554 & 2.00000000000 & 0.250000000000 & 0.375363794656605 & 85 & 18.7284343013 & 2.00000000000 & 0.250000000000 & 0.374632703499330 \\
\hline 39 & 18.7402283103 & 2.00000000000 & 0.250000000000 & 0.375340910781700 & 87 & 18.7284152965 & 2.00000000000 & 0.250000000000 & 0.374631563018360 \\
\hline 40 & 18.7399950118 & 2.00000000000 & 0.250000000000 & 0.375326893016290 & 88 & 18.7284147099 & 2.00000000000 & 0.250000000000 & 0.374631527822100 \\
\hline 41 & 18.7398545449 & 2.00000000000 & 0.250000000000 & 0.375318453215175 & 89 & 18.7283645428 & 2.00000000000 & 0.250000000000 & 0.374628517294505 \\
\hline 42 & 18.7395753099 & 2.00000000000 & 0.250000000000 & 0.375301676058585 & 90 & 18.7282463102 & 2.00000000000 & 0.250002518400 & 0.374624176059245 \\
\hline 46 & 18.7379606383 & 2.00000000000 & 0.250000000000 & 0.375204672250535 & 92 & 18.7282463102 & 2.00000000000 & 0.250000000000 & 0.374621422224295 \\
\hline 49 & 18.7378178180 & 2.00000000000 & 0.250000000000 & 0.375196092910960 & 99 & 18.7282462828 & 2.00000000000 & 0.250000000001 & 0.374621420576580 \\
\hline 50 & 18.7325416367 & 2.00000000000 & 0.250000000000 & 0.374879239803340 & 100 & 18.7282462828 & 2.00000000000 & 0.250000000001 & 0.374621420576580 \\
\hline 52 & 18.7315947043 & 2.00000000000 & 0.250000000000 & 0.374822392098025 & & & & & \\
\hline
\end{tabular}

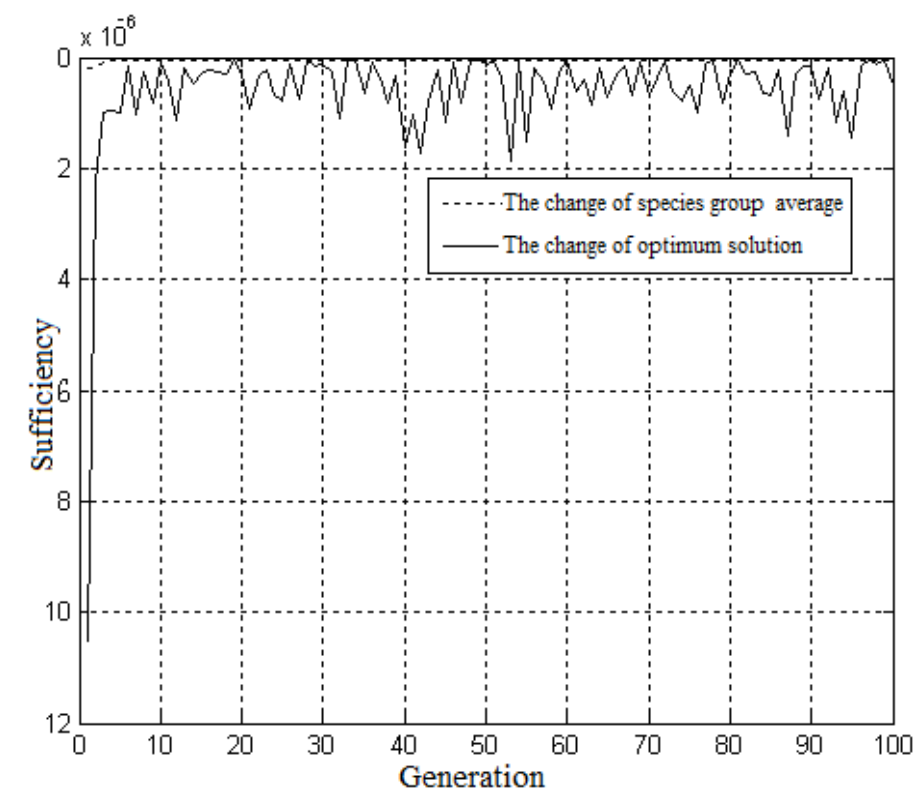

Fig. 2 Search process curve of bevel gear reliability optimum based on genetics algorithm The calculation shows that breeding to the 89th generation, the optimal solution of reliability optimum design for bevel gear driven system is obtained. The optimal solution is:

$$
\begin{aligned}
& x^{*}=\left(x_{1}^{*}, x_{2}^{*}, x_{3}^{*}\right)^{T}=(18.728,2.0,0.25)^{T} \\
& C_{T}=0.375 \mathrm{~kg}
\end{aligned}
$$


The optimum parameters of bevel gear driven system are obtained under meeting reliability constraints of Eqs.10 and 11 more than $99.99 \%$. Then, the optimum parameters are changed into integers that are final parameters of optimum design.

\section{Summary}

1) The reliability optimum design model of bevel gear driven system is established. The design parameters of system reliability optimum are obtained based on genetics algorithm.

2) The method is fit for the programming operation. The theory of reliability is introduced into the optimum design of bevel gear to provide the theoretical basis for quantification design in the gear manufacturing process.

\section{Acknowledgments}

This work was supported by The National Natural Science Foundation of China (Grant No.E050402/51105187), General Project of Education Department in Liaoning Province (Grant No.L2014110), Innovation Team Project of Colleges and Universities in Liaoning Province (Grant No. LT2015014), Natural Science Foundation in Liaoning Province (Grant No. 201602393), and Youth Science and Technology Talent Training Project in Anshan City (Grant No. 0000077). The authors would like to thank the editors and anonymous reviewers for their useful comments.

\section{References}

[1] Daxian Cheng. Hand book for mechanical design. Chemical industry press, 2014.

[2] Yimin Zhang. Reliability design for automobile parts. Beijing institute of technology press, 2000.

[3] Zhili Sun, Liangyu Chen. Practical mechanic reliability design theory and method. Science press, 2003.

[4] Chang Li, Zhili Sun. Dynamic virtual simulation of deep groove ball bearing based on elastohydrodynamic lubrication theory. Journal of Aerospace Power. 2009, 24(4):951-756.

[5] Chandhary A B. Solution method for static and dynamic analysis of three-dimensional contact problems with friction. Computer and Structure. 1986, 24(6):855-873.

[6] Liangyu Chen. A method of precise calculation of root stresses of spiral bevel gears. Chinese Journal of Mechanical Engineering. 1994, 7:4-5.

[7] Ming Yan, Zhili Sun, Qiang Yang. Analysis method of reliability sensitivity based on response surface methods. Chinese Journal of Mechanical Engineering. 2007, 43(10):67-71.

[8] Uneeb M. The effects of number ob balls and preload on vibrations associated with ball bearings. Journal of Tribology. 1997, 119(4):747-752.

[9] Haixia Du. Optimal design of bevel gear reducer based on genetic algorithm. Mechanic Manufacturing and Research. 2010(6):29-30. 